\title{
EDITORIAL
}

\section{Paediatric cardiopulmonary bypass surgery: the challenges of heterogeneity and identifying a meaningful endpoint for clinical trials}

\author{
V. J. Pappachan ${ }^{1 *}$, K. L. Brown ${ }^{2}$ and S. M. Tibby ${ }^{3}$
}

(๑) 2016 Springer-Verlag Berlin Heidelberg and ESICM

Survival following paediatric surgery for congenital heart disease (CHD) has improved dramatically over the past two decades; 30-day mortality estimates are now $2.5-4 \%$ [1]. However short- and long-term morbidity remain significant issues and hospital length of stay remains unchanged [1]. Both are costly and are linked to poorer long-term neurodevelopmental outcomes [2]. We are now operating on a greater proportion of younger, smaller patients; many with associated extra-cardiac anomalies and chromosomal disorders [3]. Ideally trials should report both immediate perioperative and postdischarge outcomes [4].

Morbidity and mortality are influenced by perioperative myocardial dysfunction. A postoperative low cardiac output syndrome (LCOS) is common, can compromise end organ function, and require inotropic and mechanical cardiovascular support including extracorporeal membrane oxygenation (ECMO). The incidence of LCOS is related to the duration of cardiopulmonary bypass (CPB), CPB-mediated inflammation, ischaemia-reperfusion injury (IRI) and the "controlled trauma" of surgery. Therefore, interventions that reduce inflammation and attenuate IRI are of potential interest. Addition of nitric oxide (NO) into the oxygenator during bypass is one such candidate. NO acts as both an antioxidant and antiinflammatory agent and has beneficial effects on cell signalling (inhibition of nuclear transcription factors-NF-K B/AP-1) [5]. NO therapy is normally delivered via inhalation and its effects have been considered to be limited

\footnotetext{
*Correspondence: jvp@soton.ac.uk

1 Paediatric Intensive Care Unit, Southampton Children's Hospital, Southampton, UK

Full author information is available at the end of the article
}

to the pulmonary circulation owing to an extremely rapid inactivation following contact with haemoglobin [6]. However, NO is now thought to remain active in the bloodstream for longer; plasma nitrosylated NO species act to conserve and transport NO, providing systemic effects [7].

In vitro models have shown that addition of $\mathrm{NO}$ to the sweep gas during CPB prevents the usual drop in cyclic guanosine monophosphate for up to $3 \mathrm{~h}$, as well as attenuating the typical fall in platelets [8]. Two small clinical studies of NO during CPB have suggested benefit with a reduction of the duration of postoperative mechanical ventilation alongside reduced serum markers of inflammation and myocardial injury $[9,10]$.

In a recent article in Intensive Care Medicine, James et al. extended these findings in a well-conducted single-centre randomised controlled trial in 198 children undergoing surgery with $\mathrm{CPB}$ [11]. The primary outcome measure was the incidence of LCOS, which the authors defined as one or more of the following: lactate greater than $4 \mathrm{mmol} / \mathrm{l}$ and $\mathrm{ScvO}_{2}$ less than $60 \%\left(\right.$ or $\mathrm{SaO}_{2}-\mathrm{ScvO}_{2}$ difference greater than $35 \%$ in a single ventricle), a vasoactive inotrope score (VIS) greater than 10 or the need for ECMO. This study demonstrated a very large effect size, with NO being associated with a $50 \%$ relative reduction in the incidence of LCOS compared to children receiving standard treatment (15\% compared to $31 \%$, $p=0.007)$. The fact that this effect size was greatest in patients less than 2 years of age has biological plausibility; the neonatal/infant myocardium is more vulnerable to reactive oxygen species [12].

Unfortunately there is no accepted standard definition of LCOS. The detail of the definition used may have

\section{国


an impact on the generalisability of the findings of this important study. Is this result 'fragile' to the definition used?

Nine patients required ECMO and in five $(55 \%)$ of these, ECMO was initiated in the operating theatre where it is often initiated because of the presence of residual anatomical lesions [13]. None of these patients met any of the authors' other predefined definitions of LCOS and therefore are unlikely to have benefited from NO therapy.

In addition the cut-off value of at least 10 for VIS was modest, and may, as the authors acknowledge, have resulted in an overdiagnosis of LCOS. For example a patient receiving $0.5 \mu \mathrm{g} / \mathrm{kg} / \mathrm{min}$ of milrinone and $5 \mu \mathrm{g} / \mathrm{kg} /$ min of dopamine would have a VIS of at least 10, which could be classed as standard postoperative support in many units. This is supported by the data provided in James et al.s supplementary Tables 2 and 4 [11], demonstrating that only $18 \%(7 / 40)$ of patients with a VIS score of at least 10 had a raised lactate and low $\mathrm{SvO}_{2}$, suggesting inadequate systemic oxygen delivery.

If the authors had selected a more stringent definition of LCOS, for example by setting the VIS threshold at 15 and excluding the patients receiving ECMO, who had no other features of LCOS, the effect size for the primary outcome (LCOS) in this study would still be clinically important: $12 \%(\mathrm{NO})$ versus $17.5 \%$ (control), with

\section{STAGE 1}

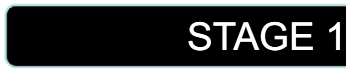

\section{STAGE 2}

\section{STAGE 3}

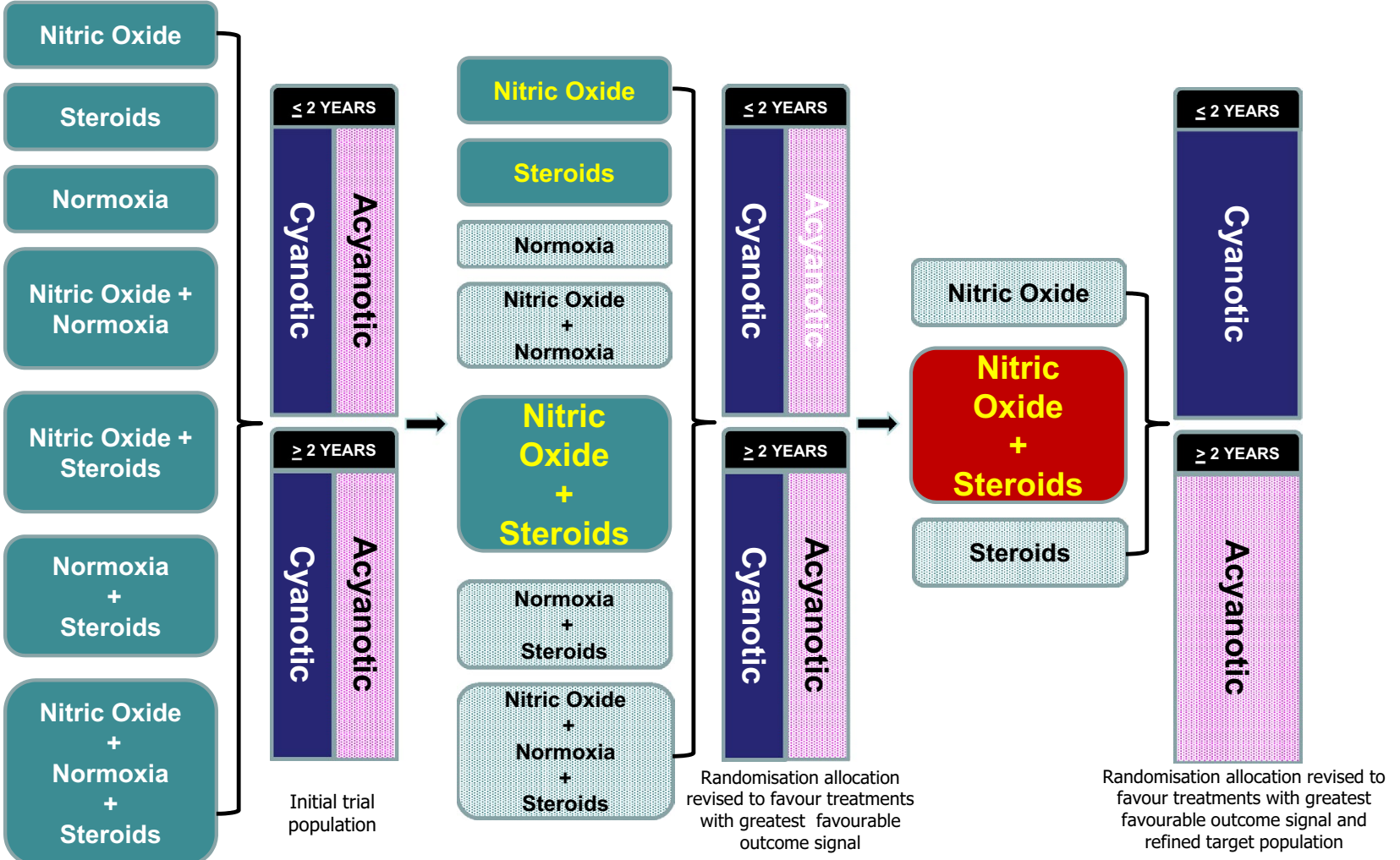

Fig. 1 A purely hypothetical example of a platform trial—no published data exist to justify the example interventions used nor their adaptive prioritisation. The platform for a trial using adaptive trial methodology would be children with congenital heart disease (CHD) undergoing surgery involving the use of cardiopulmonary bypass (CPB). This population could be stratified by age and the presence or absence of cyanotic lesions. Interventions: nitric oxide (NO) — NO at 20 ppm in the sweep gas during CPB; steroids — perioperative methylprednisolone; normoxia — the titration of oxygen concentration in the sweep during CPB to achieve normoxic as opposed to hyperoxic conditions. These are all interventions that have been tested with variable outcome benefit in small populations using traditional single-disease, single-treatment trial design. This figure describes a hypothetical model in which adaptive trial methodology could be applied to this platform. In the first stage interventions are applied individually and in all possible combinations, in a randomised fashion using equal allocation. The results of the hypothetical first outcome analysis suggest that there is a strong signal with $\mathrm{NO}+$ steroids (NOS) and a moderate signal with $\mathrm{NO}$ and steroids (S) when used as isolated interventions. In the second hypothetical stage therefore, although all treatment combinations are tested once again, the randomisation uses unequal allocation to favour those treatment combinations with the strongest outcome signal (NOS > NO = S). During hypothetical stage 2 it becomes clear that there is a negligible outcome signal in acyanotic children $\leq 2$ years of age or in cyanotic children $\geq 2$ years of age and that the favourable outcome signal is very much stronger in the group receiving NOS. Therefore in hypothetical stage 3 , randomisation again using unequal allocation (NOS $\gg$ NO $=\mathrm{S}$ ) is used to test only these three interventions in cyanotic children $\leq 2$ years of age and in acyanotic children $\geq 2$ years of age 
a relative risk reduction of $31 \%$. However using conventional indices of inadequate systemic delivery (high lactate and a low $\mathrm{SvO}_{2}$ ), would have led to only $4 \%$ of the patients in the NO group and 3\% of controls being defined as having LCOS. This, and the lack of any significant effect on any of the more standard clinical outcomes, emphasises the inadequacy of our present definition of LCOS.

One of the challenges in evaluating new therapies via the "traditional" two-arm multi-centre superiority trial in paediatric $\mathrm{CPB}$ is the significant inter- and intra-hospital heterogeneity in practice. Improvements in clinical outcomes may require modulation of several aspects of the care pathway and a re-evaluation of appropriate outcome measures. Multiple interventions delivered individually/ in combination may need to be tested $\mathrm{NO}$, the target partial pressure of oxygen on bypass, the use of perioperative steroids being three examples). A recent article by Berry et al. [14], presenting an overview of platform trial design using adaptive trial methodology, is of particular relevance. The goal of a platform trial is to identify superior combinations of therapies for a given disease by using specialised statistical tools to both allocate patients and analyse outcomes. As data accumulates within such a trial, allocation to randomised treatments are adjusted to preferentially expose the platform to the intervention with the greatest positive outcome signal. A hypothetical example of such a trial is shown in Fig. 1. An early example of this approach was used in one of the first trials of paediatric ECMO referred to as the 'play the winner trial' [15] and it provides an interesting option for a future trials in paediatric cardiac surgery. One of the priorities of such a platform trial might be to define, consolidate and validate a definition of LCOS and relate this to clinically relevant short- and long-term outcomes.

\footnotetext{
Author details

1 Paediatric Intensive Care Unit, Southampton Children's Hospital, Southampton, UK. ${ }^{2}$ Great Ormond Street Hospital NHS Trust, London, UK. ${ }^{3}$ Evelina
} London Children's Hospital, London, UK.

\section{Compliance with ethical standards}

\section{Conflicts of interest}

The authors declare that they have no competing interests..

Received: 31 October 2016 Accepted: 1 November 2016 Published online: 18 November 2016
References

1. Jacobs JP, He X, Mayer JE et al (2016) Mortality trends in pediatric and congenital heart surgery: an analysis of the Society of Thoracic Surgeons Congenital Heart Surgery Database. Ann Thorac Surg 102:1345-1352. doi:10.1016/j.athoracsur.2016.01.071

2. Newburger JW, Sleeper LA, Bellinger DC et al (2012) Early developmental outcome in children with hypoplastic left heart syndrome and related anomalies: the single ventricle reconstruction trial. Circulation 125:2081-2091. doi:10.1161/CIRCULATIONAHA.111.064113

3. Brown KL, Crowe S, Franklin R et al (2015) Trends in 30-day mortality rate and case mix for paediatric cardiac surgery in the UK between 2000 and 2010. Open Heart 2:e000157. doi:10.1136/openhrt-2014-000157

4. Brown KL, Wray J, Knowles RL et al (2016) Infant deaths in the UK community following successful cardiac surgery: building the evidence base for optimal surveillance, a mixed-methods study. NIHR J Libr, Southampton (UK)

5. Guzik TJ, Korbut R, Adamek-Guzik T (2003) Nitric oxide and superoxide in inflammation and immune regulation. J Physiol Pharmacol 54:469-487

6. Liao JC, Hein TW, Vaughn MW et al (1999) Intravascular flow decreases erythrocyte consumption of nitric oxide. Proc Natl Acad Sci USA 96:8757-8761

7. Rassaf T, Kleinbongard P, Kelm M (2005) Circulating NO pool in humans. Kidney Blood Press Res 28:341-348. doi:10.1159/000090188

8. Mellgren K, Friberg LG, Mellgren G et al (1996) Nitric oxide in the oxygenator sweep gas reduces platelet activation during experimental perfusion. Ann Thorac Surg 61:1194-1198

9. Gianetti J, Del Sarto P, Bevilacqua S et al (2004) Supplemental nitric oxide and its effect on myocardial injury and function in patients undergoing cardiac surgery with extracorporeal circulation. J Thorac Cardiovasc Surg 127:44-50. doi:10.1016/j.jtcvs.2002.08.001

10. Checchia PA, Bronicki RA, Muenzer JT et al (2013) Nitric oxide delivery during cardiopulmonary bypass reduces postoperative morbidity in children-a randomized trial. J Thorac Cardiovasc Surg 146:530-536. doi:10.1016/j.jtcvs.2012.09.100

11. James C, Millar J, Horton S et al (2016) Nitric oxide administration during paediatric cardiopulmonary bypass: a randomised controlled trial. Intensive Care Med 42:1744-1752. doi:10.1007/s00134-016-4420-6

12. Fudulu D, Angelini G (2016) Oxidative stress after surgery on the immature heart. Oxid Med Cell Longev 2016:1971452. doi:10.1155/2016/1971452

13. Agarwal HS, Hardison DC, Saville BR et al (2014) Residual lesions in postoperative pediatric cardiac surgery patients receiving extracorporeal membrane oxygenation support. J Thorac Cardiovasc Surg 147:434-441. doi:10.1016/j.jtcvs.2013.03.021

14. Berry SM, Connor JT, Lewis RJ (2015) The platform trial: an efficient strategy for evaluating multiple treatments. JAMA 313:1619-1620. doi:10.1001/jama.2015.2316

15. Bartlett RH, Roloff DW, Cornell RG et al (1985) Extracorporeal circulation in neonatal respiratory failure: a prospective randomized study. Pediatrics $76: 479-487$ 